\title{
Malignant Ventricular Arrhythmic Storm Triggered by Short-coupled Premature Ventricular Contractions Arising from the Anterolateral Papillary Muscle
}

\author{
Peter Kabunga, ${ }^{1}$ Caroline Medi, ${ }^{1}$ Laura Yeates ${ }^{2}$ and Raymond W Sy ${ }^{1,3}$ \\ 1. Department of Cardiology, Royal Prince Alfred Hospital, New South Wales, Australia; 2. Agnes Ginges centre for Molecular Cardiology, Centenary Institute, \\ New South Wales, Australia; 3. Sydney Medical School, University of Sydney, New South Wales, Australia.
}

DOI: http://doi.org/10.17925/EJAE.2016.02.01.33

\begin{abstract}
A case of idiopathic ventricular fibrillation (VF), triggered by ectopic beats originating from the anterolateral papillary muscle, is presented. The arrhythmia was characterised by short-coupled premature ventricular contractions (PVCs), which were resistant to isoproterenol, and ultimately treated with catheter ablation.
\end{abstract}

\section{Keywords}

Idiopathic ventricular fibrillation, papillary muscle, isoproterenol

Disclosure: Peter Kabunga, Caroline Medi, Laura Yeates and Raymond W Sy have nothing to disclose in relation to this article. No funding was received in the publication of this article.

Open Access: This article is published under the Creative Commons Attribution Noncommercial License, which permits any noncommercial use, distribution, adaptation, and reproduction provided the original author(s) and source are given appropriate credit.

Compliance with Ethics: All procedures were followed in accordance with the responsible committee on human experimentation and with the Helsinki Declaration of 1975 and subsequent revisions, and informed consent was received from the patient involved in this case study.

Received: 22 February 2016 Accepted: 4 April 2016 Citation: European Journal of Arrhythmia \& Electrophysiology, 2016;2(1):33-6

Correspondence: Raymond W Sy, Department of Cardiology, Royal Prince Alfred Hospital, Camperdown, New South Wales 2050, Australia. E: raymond.sy01@gmail.com

Supplementary Information: An accompanying video to this article can be found at

www.touchcardio.com/gallery/malignant-ventricular-arrhythmic-storm-triggered-short-coupled-premature-ventricular

Premature ventricular contractions (PVCS) arising from the Purkinje network are rare causes of idiopathic ventricular fibrillation (IVF). ${ }^{1-4}$ Van Herendael and colleagues recently highlighted the importance of triggers from the papillary muscles (PM) and the left ventricular outflow tract in the initiation of ventricular fibrillation. ${ }^{4}$ In their study, eight of 30 patients had PM triggers which were successfully targeted with catheter ablation. Although acute success was reported in all PM-mediated ventricular tachycardia (VT)/ventricular fibrillation (VF) patients, 38\% of patients had a recurrence of PVCs during long-term monitoring, perhaps due to poor catheter stability and presumed intramurality of target sites.

A range of medical therapies - including beta-blockers, amiodarone, verapamil and quinidine - have been variably described in the longterm management of idiopathic VF. In addition, isoproterenol has been acutely used in the setting of idiopathic VF storms associated with early repolarisation syndromes. ${ }^{5-7}$ However, there are few reports regarding the specific response of PM-mediated VT/VF to pharmacological therapy.

We report a case of a malignant, isoproterenol-resistantVF storm, triggered by short-coupled monomorphic PVCs originating from the anterolateral papillary muscle (APM) successfully treated with catheter ablation.

\section{Case report}

A 47-year-old woman presented to her local hospital with recurrent nocturnal seizures. Cardiac monitoring revealed non-sustained polymorphic ventricular tachycardia and ventricular fibrillation preceding seizure activity. Over the ensuing 24 hours, she received 10 shocks, despite a sequential trial of pharmacological therapy with intravenous (IV) amiodarone (300 mg bolus plus $900 \mathrm{mg}$ continuous infusion), $40 \mathrm{mmol}$ of magnesium sulphate $\left(\mathrm{MgSO}_{4}\right), 40 \mathrm{mmol}$ of potassium chloride $(\mathrm{KCl})$ and boluses of IV metoprolol. Isoproterenol was then administered at the peripheral hospital as she continued to get multiple PVCS and non-sustained VT and the patient was transferred to our centre for further evaluation.

She had been previously treated with carbamazepine and valproate for 10 years, with the presumed diagnosis of epilepsy. Notably, the patient's symptoms pre-dated the prescription of anti-epileptic medications. There was also a family history of premature sudden cardiac death (Figure 1).

Baseline investigations including electrolytes, trans-thoracic echocardiogram (TTE), and electrocardiogram (ECG) (including QT interval) during sinus rhythm were normal. The QRS complexes in leads V1-V3 remained normal in all ECGs, and there was no J-point elevation observed before or after episodes of arrhythmia (Figure 2). Episodes of sustained ventricular arrhythmia appeared to be consistently triggered by monomorphic PVCS with a short coupling interval (240 ms) (Figure 3). Interestingly, we did not observe a critical difference in the coupling intervals between isolated PVCs and those PVCs initiating torsades de pointes/VF (Figures 2 and 3). Coronary angiography, cardiac magnetic resonance, flecainide challenge and signal averaged ECG, were normal. 


\section{Figure 1: Family pedigree and baseline electrocardiogram}

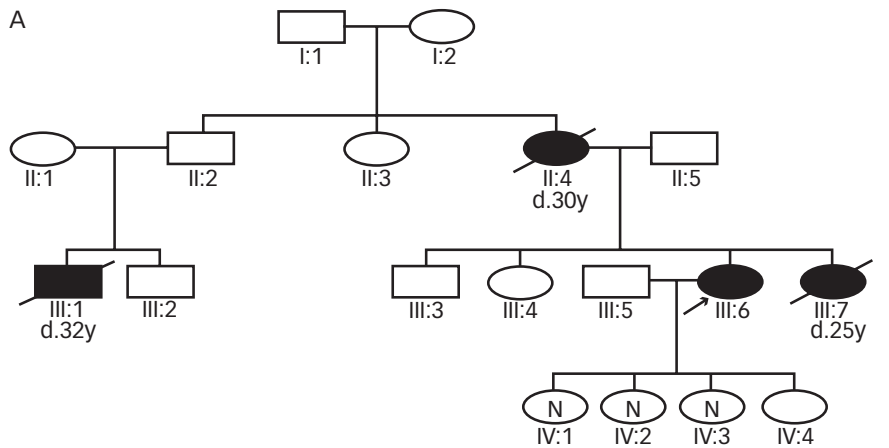

B
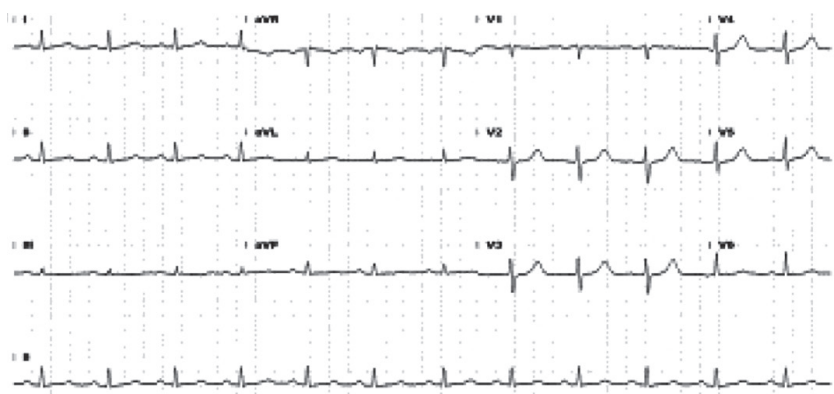

A) Family pedigree showing three unexplained deaths before the age of 35 years. squares = male; circles = female; line through symbol = deceased individual, arrow indicates proband, filled-in symbol = sudden cardiac death $/ V F$, open symbol with $N$ = normal electrocardiogram and echocardiogram; open symbols = unknown clinical status, $d$. age = age at death in years. B) 12-lead electrocardiogram with no premature ventricular contractions.

\section{Figure 2: Short-coupled premature ventricular contraction initiating ventricular fibrillation}

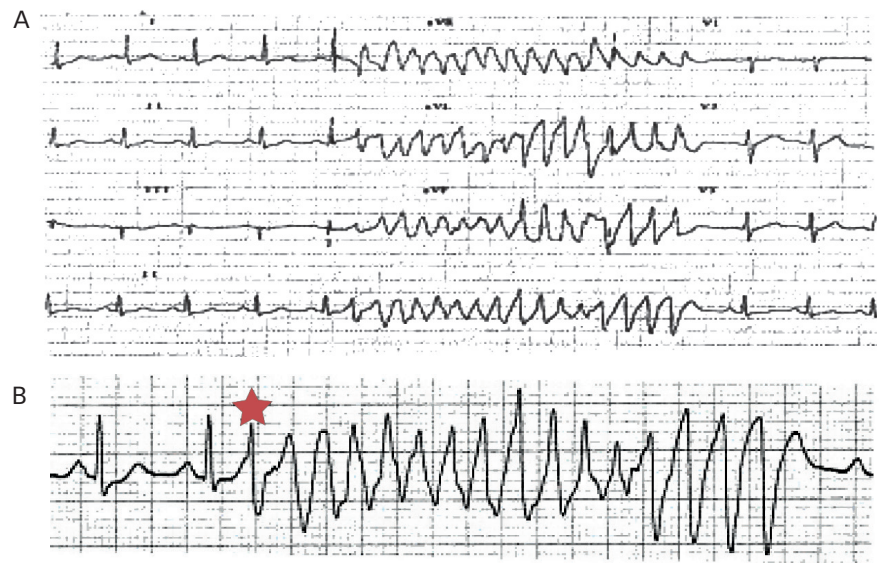

Initiating premature ventricular contractions (PVCS) marked with red star

Even though there was no definite evidence of early repolarisation syndrome, isoproterenol (2 $\mathrm{mcg} / \mathrm{min}$ ) was empirically continued in an attempt to suppress VF. However, isoproterenol was associated with an abrupt increase in the frequency of PVCs and non-sustained ventricular tachycardia (NSVT) and it was ceased. Intravenous esmolol (2.5 g infusion) suppressed the NSVT but isolated breakthrough PVCS remained. An electrophysiology (EP) study was performed due to persistence of short-coupled PVCs (Figure 2).

The EP study was performed with minimal sedation. Baseline EP was unremarkable with normal baseline intervals (QTC, AH, HV). Clinical PVCs were infrequently observed at baseline. Ventricular effective refractory period was $230 \mathrm{~ms}$. Ventricular arrhythmias were not inducible with burst pacing or programmed electrical stimulation (PES) with up to four extra-stimuli delivered from the right ventricular apex to refractoriness using two drive cycles (600 ms, $400 \mathrm{~ms}$ ). Electro-anatomical mapping (Carto-3, Biosense-Webster) of the left ventricle was performed using a combined retro-aortic and transseptal approach. Unipolar and bipolar voltage maps of the left ventricle were normal. Activation mapping was not feasible due to paucity of PVCs at baseline and with Isoproterenol (up to $5 \mathrm{mcg} / \mathrm{min}$ ). There were subtle differences in the morphology of the spontaneous PVCs at the time of the EP study when compared to the PVCs previously captured on the ward, but this was probably related to differences in lead positioning. The PVC morphology was suggestive of an origin in the mid-anterolateral LV and detailed pace-mapping was performed in this region. Subtle differences in the morphology of the QRS complex were observed when pacing at different sites along the anterolateral papillary muscle (e.g. base versus apex, anterior versus posterior). The best pace-map appeared to be reproducible when pacing at low output within a relatively small area of the papillary muscle. However, this observation was limited by catheter movement related to cardio-respiratory motion, and the spatial resolution of the electroanatomical mapping system and real-time echocardiography (Figure 4 and video). TTE was used instead of intra-cardiac echocardiography (ICE) in this case because the latter was not immediately available given the emergent nature of the procedure. There appeared to be latency ( $20 \mathrm{~ms}$ ) between the pacing stimulus and the onset of the surface QRS. At the onset of radiofrequency (RF) applications (irrigated 35 Watts, 45 C), PVC automaticity matching the clinical template was seen (Figure 3). Following this, no further spontaneous PVCs or ventricular arrhythmias were observed. In addition, PES using up to four extra-stimuli as well as burst pacing delivered from the right ventricular apex and the left ventricle did not induce any ventricular arrhythmias either from the target or remote sites. No PVCs were seen during extended in-hospital telemetry over the ensuing seven days. There was also no evidence of inducible ventricular ectopy on adrenaline challenge or exercise testing. A single chamber implantable cardioverter-defibrillator was implanted and the patient remains arrhythmia free with a very low PVC count, off medications at 17 months follow-up.

\section{Discussion}

The diagnosis in this case was a variant of short-coupled idiopathic ventricular fibrillation, triggered by PVCs originating from the papillary muscle. The case highlights the malignant nature of this arrhythmia, and the challenges in its management including the role of ablation.

Transmural dispersion of repolarisation is thought to predispose the ventricular myocardium to phase two re-entry-mediated VF following excitation by short-coupled PVCs. ${ }^{8}$ Isoproterenol reverses this repolarisation abnormality by homogenising transmural and epicardial electrical gradients, and is effective in terminating idiopathic VF (IVF) associated with early repolarisation (ER). ${ }^{6.8,9}$ Our patient had no obvious ER and she experienced an increase in PVC and NSVT burden with isoproterenol and improvement with esmolol, perhaps indicating that phase two re-entry was not the underlying mechanism of VF initiation. Because of a low baseline blood pressure, she was prescribed a small of dose of metoprolol (25 mg twice daily), which she voluntary ceased during follow-up. Improvement with beta-blockers and an increase in short coupled PVCs with Isoproterenol is more typical for cyclic AMPmediated triggered activity seen in the rare, malignant forms of outflow tract ventricular arrhythmias. ${ }^{10,11}$ Beta-blockers are usually effective because they inhibit adenylate cyclase, which leads to a decrease 


\section{Figure 3: Twelve-lead electrocardiogram with multiple short-coupled premature ventricular contractions $(A)$ and repeated ventricular fibrillation initiation with short-coupled premature ventricular contractions (B)}

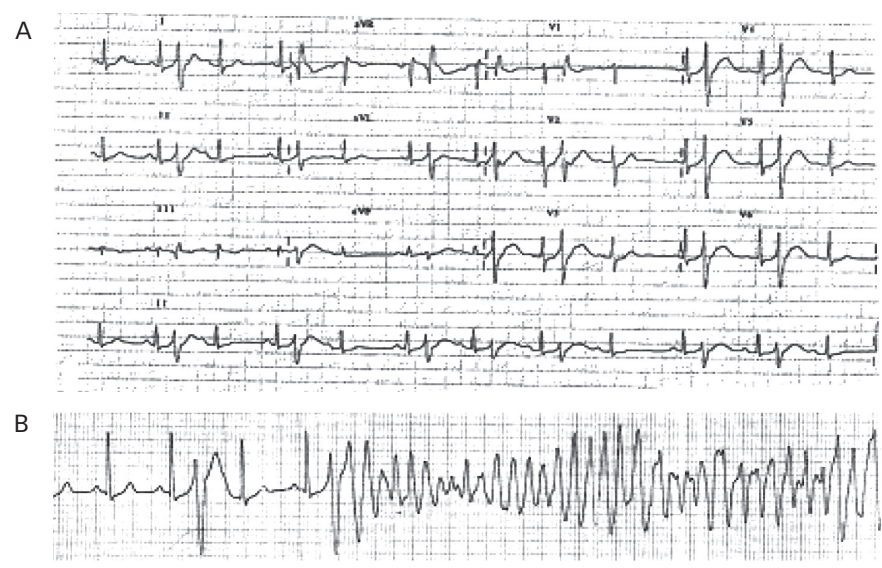

of CAMP and intracellular calcium, preventing the onset of delayed after-depolarisations typical for triggered arrhythmias such as outflow tract $\mathrm{VT} .^{12}$ It remains speculative whether sensitivity to beta-adrenergic stimulation is a unique feature of ectopy from the papillary muscle but the present case raises the possibility that isoproterenol may aggravate rather than ameliorate this specific type of IVF, especially in the absence of ER. However, J-point elevation is variable and in a small number of patients with proven ER-associated IVF, may be absent at the time of presentation. . $13-17^{17}$

Leenhardt et al. have previously described a short-coupled variant of idiopathic VF presenting with torsade de pointes. ${ }^{18}$ In their study of 14 patients with apparently normal baseline ECGs, ventricular arrhythmia was induced with PES in only one patient. Response to isoproterenol was variable, with suppression of PVCs in three patients, no effect in six patients, and an increase in two patients. They reported the efficacy of verapamil but not beta-blockers, in some patients. However, verapamil did not universally protect against sudden death, highlighting the need for implantable cardioverter defibrillator (ICD) therapy. Kondo et al. have also recently described a 19-year-old patient with short-coupled PVCs precipitating VF. ${ }^{19}$ Interestingly, their patient had inferolateral ER but isoproterenol infusion increased PVCS, while verapamil and propranolol infusion suppressed them. Clinical PVCs were not elicited with PES and catheter ablation at a site in the right ventricular Purkinje system successfully terminated the PVCS. Isoproterenol-resistant PVCS triggering recurrent VF has also been described in a patient with Brugada syndrome (BrS), suggesting a rare subset of patients with BrS/ IVF exists with non-phase two-mediated re-entry as a mechanism for ventricular arrhythmia. ${ }^{20}$ Given the apparent response to intravenous

\section{Figure 4: Catheter ablation of ventricular ectopy}

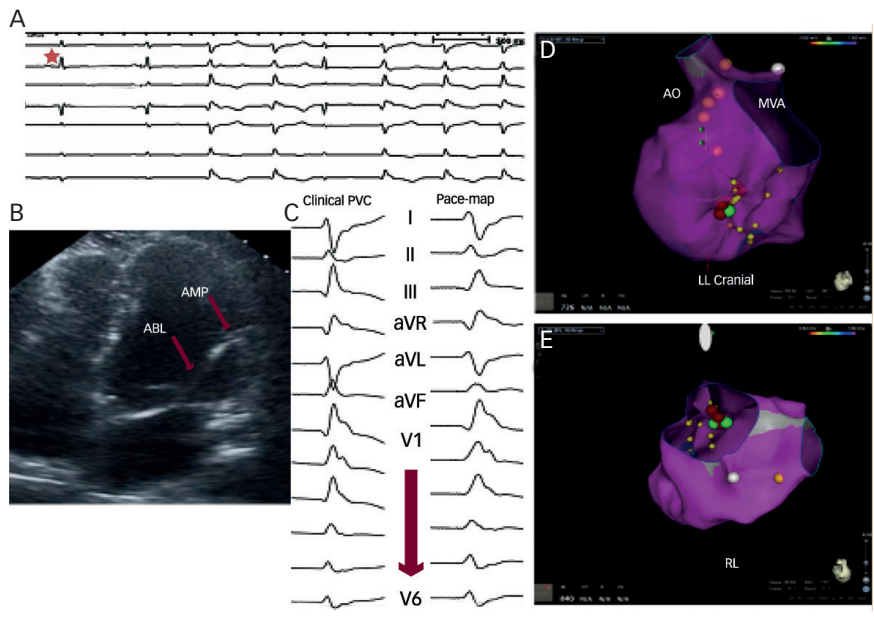

Clinical premature ventricular contraction automaticity following onset of radiofrequency delivery (A) onset of ablation has been annotated with a star; intra-procedural transthoracic echo showing tip of ablation catheter at apex of the anterolateral papillary muscle (apical four-chamber view) (B); pace-map of effective ablation site (C) and electro-anatomical mapping images of the successful ablation site at anterolateral papillary muscle (D,E). $A B L=$ ablation catheter; $A P M=$ anterolateral papilary muscle; $M V A=$ mitral valve annulus; $A O=$ aorta; $L L=$ left lateral; $R L=$ right lateral; red star = ablation on

esmolol in the present case, beta-blockade may be considered as a viable long-term therapeutic strategy for this specific subset of idiopathic VF, but it is important to note that the acute response was incomplete suppression.

It is likely that there may be a genetic predisposition to ventricular arrhythmia in our patient given the family history of three sudden unexplained deaths before the age of 35 years (Figure 1). Clinical genetic testing was considered but ultimately deferred in the absence of a clear phenotype for one of the recognised channelopathies. Research genetic testing, such as exome sequencing, has been discussed with the patient and her family but has also been deferred until phenotyping of the entire family has been confirmed to facilitate co-segregation studies.

Catheter ablation of focal papillary muscle triggers is challenging and associated with a high recurrence rate. ${ }^{8,21}$ This is thought to be related to difficulties in visualising the papillary muscles, achieving optimal catheter stability and an inability to deliver transmural lesions. Previous reports advocate the use of irrigated RF and ICE to improve outcomes. ${ }^{8,21}$ In laboratories where it is readily available, ICE offers advantages over standard TTE with improved resolution, minimisation of acoustic shadowing, and potential for integration with some electroanatomical mapping systems. In our case, an irrigated tip catheter with contact-force sensing technology, electroanatomical mapping and real-time ultrasound were useful to facilitate successful ablation.

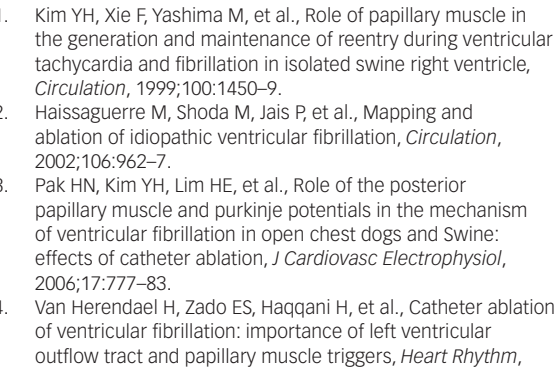

2014;11:566-73.

Mittadodla PS, Salen PN, Traub DM, Isoproterenol as an adjunct for treatment of idiopathic ventricular fibrillation storm in a pregnant woman, Am J Emerg Med, 2012;30:251.e3-5. Aizawa Y Chinushi M, Hasegawa K, et al. Electrical storm in idiopathic ventricular fibrillation is associated with early repolarization, J Am Coll Cardiol, 2013:62:1015-9. repolarization, J Am Coll Cardiol, 2013;62:1015-9.
Kasanuki H, Ohnishi S, Ohtuka M, et al., Idiopathic ventricular Kasanuki $\mathrm{H}$, Ohnishi S, Ohtuka M, et al., Idiopathic ventricu
fibrillation induced with vagal activity in patients without fibrillation induced with vagal activity in patients without obvious heart disease, Circulation, 1997;95:2277-85.
Koncz I, Gurabi Z, Patocskai B, et al., Mechanisms underlyin the development of the electrocardiographic and arrhythmic manifestations of early repolarization syndrome, $\mathrm{J} \mathrm{Mol} \mathrm{Cell}$ Cardiol, 2014;68:20-8.

Haissaguerre M, Sacher F, Nogami A, et al., Characteristics of recurrent ventricular fibrillation associated with inferolateral early repolarization role of drug therapy, I Am Coll Cardiol, 2009:53:612-9.

10. Shimizu W, Arrhythmias originating from the right ventricular outflow tract: how to distinguish "malignant" from "benign"? Heart Rhythm, 2009:6:1507-11.

11. Viskin $S$, Rosso R, Rogowski O, Belhassen B, The "shortcoupled" variant of right ventricular outflow ventricular tachycardia: a not-so-benign form of benign ventricular tachycardia?, J Cardiovasc Electrophysiol, 2005;16:912-6.

12. Lerman BB, Belardinelli L, West GA, et al., Adenosine-sensitive ventricular tachycardia: evidence suggesting cyclic AMPmediated triggered activity, Circulation, 1986;74:270-80

13. Haissaguerre M, Derval N, Sacher F, et al., Sudden cardiac arrest associated with early repolarization, N Eng/ । Med, 
2008;358:2016-23.

14. Aizawa $\mathrm{Y}$, Sato $\mathrm{A}$, Watanabe $\mathrm{H}$ et al., Dynamicity of the J-wave in idiopathic ventricular fibrillation with a special reference to pause-dependent augmentation of the J-wave, J Am Coll Cardiol, 2012;59:1948-53.

15. Shinohara T, Takahashi N, Saikawa T, Yoshimatsu H, Characterization of J wave in a patient with idiopathic ventricular fibrillation, Heart Rhythm, 2006;3:1082-4.

16. Nam GB, Kim YH, Antzelevitch C, Augmentation of J waves and electrical storms in patients with early repolarization,
N Engl J Med, 2008;358:2078-9

17. Nam GB, Ko KH, Kim J, et al., Mode of onset of ventricular fibrillation in patients with early repolarization pattern vs. Brugada syndrome, Eur Heart J, 2010;31:330-9.

18. Leenhardt A, Glaser E, Burguera M, et al., Short-coupled variant of torsade de pointes. A new electrocardiographic entity in the spectrum of idiopathic ventricular
tachyarrhythmias, circulation, 1994:89:206-15.

19. Kondo $\mathrm{H}$, Shinohara T, Takahashi $\mathrm{N}$, A case of short-coupled premature ventricular beat-induced ventricular fibrillation with early repolarization in the inferolateral leads, Journal of Arrhythmia, 2015:31:60-3.

20. Chinushi M, lijima K, Sato A, Furushima H, Short-coupling premature ventricular complexes from the left ventricle triggered isoproterenol-resistant electrical storm in a patient with Brugada syndrome, Heart Rhythm, 2013;10:916-20.

21. Good E, Desjardins B, Jongnarangsin K, et al., Ventricular arrhythmias originating from a papillary muscle in patients without prior infarction: a comparison with fascicular arrhythmias, Heart Rhythm, 2008:5:1530-7. 